PROCEEDINGS OF THE

AMERICAN MATHEMATICAL SOCIETY

Volume 125, Number 6, June 1997, Pages 1811-1814

S 0002-9939(97)03770-2

\title{
ON THE CONCEPT OF $\Pi_{1}^{1}$-COMPLETENESS
}

\author{
ALEXANDER S. KECHRIS
}

(Communicated by Andreas R. Blass)

\begin{abstract}
It is shown that two natural notions of completeness for co-analytic sets in Polish spaces, one in terms of continuous reductions and the other in terms of Borel reductions, coincide. The proof uses methods of effective descriptive set theory.
\end{abstract}

The concept of completeness of sets in various classes in the projective and other hierarchies plays a central role in descriptive set theory. A complete set in a given class is a universal object that encapsulates the complexity of the class. The concept of completeness depends a priori on the choice of a collection of "reducing" maps and the main purpose of this paper is to show that, in certain standard cases, it is actually independent of such a choice and therefore has an intrinsic character. We explain below the precise results.

Consider sets in Polish (separable completely metrizable topological) spaces. Let $\mathcal{F}$ be a class of functions between such spaces, like, for example, continuous, Borel, etc. A set $A \subseteq X, X$ a Polish space, is called $\mathcal{F} \boldsymbol{\Pi}_{1}^{1}$-hard if for every zerodimensional (i.e., having a basis consisting of clopen sets) Polish space $Y$ and every $\Pi_{1}^{1}$-set $B \subseteq Y$ there is a function $f: Y \rightarrow X, f \in \mathcal{F}$, with $B=f^{-1}[A]$. (Such an $f$ is said to reduce $B$ to $A$.) If $A$ is also $\Pi_{1}^{1}$, we call $A \mathcal{F} \boldsymbol{\Pi}_{1}^{1}$-complete. For the importance of these notions in descriptive set theory we refer to [2]. There are two natural extremes for a choice of $\mathcal{F}$ in this context, namely $\mathcal{F}_{0}=$ continuous and $\mathcal{F}_{1}=$ Borel. In the first case we simply use the terms $\boldsymbol{\Pi}_{1}^{1}$-hard, $\boldsymbol{\Pi}_{1}^{1}$-complete and in the second the terms Borel $\boldsymbol{\Pi}_{1}^{1}$-hard and Borel $\boldsymbol{\Pi}_{1}^{1}$-complete. Clearly any set which is $\boldsymbol{\Pi}_{1}^{1}$-hard (complete) is also Borel $\boldsymbol{\Pi}_{1}^{1}$-hard (complete). The question of whether the converse also holds is a natural question that often comes up. It was brought to our attention by R. Dougherty. We show here, using the method of Harrington-Kechris [1], that this is indeed the case, and therefore the notion of $\boldsymbol{\Pi}_{1}^{1}$-completeness has an intrinsic character independent of the choice of the class of reducing functions. It is perhaps worth pointing out that the method of [1] makes use of effective descriptive set theory, while the result proved deals purely with classical notions. We do not know a proof of this result using only classical methods.

Theorem. Let $X$ be a Polish space and $A \subseteq X$. Then $A$ is $\Pi_{1}^{1}$-hard (resp., complete) iff $A$ is Borel $\Pi_{1}^{1}$-hard (resp., complete).

Received by the editors October 2, 1995 and, in revised form, January 15, 1996.

1991 Mathematics Subject Classification. Primary 03E15, 04A15, 28A05, 54H05.

The author's research was partially supported by NSF Grant DMS-9317509.

(C)1997 American Mathematical Society 
Proof. First we argue that it is enough to prove the result for $X=\mathbb{N}^{\mathbb{N}}=$ the Baire space. Indeed, assume the result has been proved in this case. Let $X$ be any Polish space and $A \subseteq X$ a Borel $\Pi_{1}^{1}$-hard set. Since $X$ is Polish, fix a closed set $F \subseteq \mathbb{N}^{\mathbb{N}}$ and a continuous bijection $\pi: F \rightarrow X$. Let $\rho$ be the inverse of $X$. Then $\rho$ is Borel. Let $\sigma$ be a continuous retraction from $\mathbb{N}^{\mathbb{N}}$ to $F$, and let $A^{\prime}=(\pi \circ \sigma)^{-1}[A] \subseteq \mathbb{N}^{\mathbb{N}}$.

Since $A$ is Borel $\Pi_{1}^{1}$-hard, then (using $\rho$ ) $A^{\prime}$ is Borel $\Pi_{1}^{1}$-hard, so by our assumption $A^{\prime}$ is $\boldsymbol{\Pi}_{1}^{1}$-hard, so (using $\pi \circ \sigma$ ) $A$ is $\boldsymbol{\Pi}_{1}^{1}$-hard. (We thank the referee for suggesting this argument.)

So assume $A \subseteq \mathbb{N}^{\mathbb{N}}$ is Borel $\Pi_{1}^{1}$-hard. Let $B \subseteq \mathbb{N}^{\mathbb{N}}$ be a $\Pi_{1}^{1}$-set. We will find a continuous $f: \mathbb{N}^{\mathbb{N}} \rightarrow \mathbb{N}^{\mathbb{N}}$ with $f^{-1}[A]=B$. Since every zero-dimensional Polish space is a closed subspace of $\mathbb{N}^{\mathbb{N}}$ (see $[2,7.8]$ ) this shows that $A$ is $\boldsymbol{\Pi}_{1}^{1}$-hard.

We first review some concepts and facts from [1].

A coding system for perfect binary trees is a set $P \subseteq 2^{\mathbb{N}}$ and a surjection $\mathcal{P}: P \rightarrow$ $\{T: T$ is a perfect binary tree $\}$. For $a \in P$, let $\mathcal{P}(a) \equiv \mathcal{P}_{a}$ (= the tree coded by $a$ ). For $c \in \mathcal{P}_{a}$ let $a^{*}(c)=$ the element of $2^{\mathbb{N}}$ corresponding to $c$ under the canonical homeomorphism $a^{*}:\left[\mathcal{P}_{a}\right] \rightarrow 2^{\mathbb{N}}$ (here $[T]$ is the set of infinite branches through $T$ ). Call $\langle a, c\rangle$ good if $a \in P \& c \in\left[\mathcal{P}_{a}\right]$. We call a coding system $\langle P, \mathcal{P}\rangle$ nice if:

(i) For any $H: 2^{\mathbb{N}} \times 2^{\mathbb{N}} \rightarrow \mathbb{N}$ Borel, there is $a \in P$ and $n \in \mathbb{N}$ such that

$$
\forall c \in\left[\mathcal{P}_{a}\right](H(a, c)=n) .
$$

(ii) $P$ is $\Pi_{1}^{1}$ and for $a \in P$ the relation

$$
\mathcal{P}(s, a) \Leftrightarrow s \in \mathcal{P}_{a}
$$

is $\Delta_{1}^{1}$, i.e., there are relations $\Pi, \Sigma$ in $\Pi_{1}^{1}, \Sigma_{1}^{1}$ resp., such that for $a \in P$ :

$$
\mathcal{P}(s, a) \Leftrightarrow \Pi(s, a) \Leftrightarrow \Sigma(s, a) .
$$

Lemma ([1, 1.4.2]). There exists a nice coding system.

Proof. Apply 1.4.2 of [1] with $W=\mathbb{N}, \sim$ the equality relation on $\mathbb{N}$.

Fix such a system $(P, \mathcal{P})$ from now on.

Let $\langle\cdot, \cdot, \cdot\rangle,\langle\cdot, \cdot\rangle$ be recursive homeomorphisms of $2^{\mathbb{N}} \times 2^{\mathbb{N}} \times 2^{\mathbb{N}}, 2^{\mathbb{N}} \times 2^{\mathbb{N}}$ with $2^{\mathbb{N}}$. For any given $\left\langle a^{0}, c^{0}\right\rangle$ inductively define $\left\langle m_{i}, a^{i+1}, c^{i+1}\right\rangle(i \geq 0)$ as follows: If $\left\langle a^{i}, c^{i}\right\rangle$ is given and is good, let $\left\langle x_{i}, a^{i+1}, c^{i+1}\right\rangle=\left(a^{i}\right)^{*}\left(c_{i}\right)$. Put $m_{i}=$ the location of the first 0 in $x_{i}$, if such exists; else $m_{i}=0$. If $\left\langle a^{i}, c^{i}\right\rangle$ is not good, put $\left\langle m_{i}, a^{i+1}, c^{i+1}\right\rangle=$ $\langle 0, \overline{0}, \overline{0}\rangle$, where $\overline{0}=(0,0, \ldots)$.

Now define $B^{*} \subseteq 2^{\mathbb{N}}$ as follows:

$$
\left\langle a^{0}, c^{0}\right\rangle \in B^{*} \Leftrightarrow \forall i\left(\left\langle a^{i}, c^{i}\right\rangle \text { is good }\right) \&\left(m_{i}\right) \in B .
$$

Clearly $B^{*}$ is $\Pi_{1}^{1}$. So there is $G: 2^{\mathbb{N}} \rightarrow \mathbb{N}^{\mathbb{N}}$ Borel such that

$$
\left\langle a^{0}, c^{0}\right\rangle \in B^{*} \Leftrightarrow G\left(\left\langle a^{0}, c^{0}\right\rangle\right) \in A .
$$

Next we will find $F: \mathbb{N}<\mathbb{N} \rightarrow\left(2^{\mathbb{N}} \times \mathbb{N}\right)^{<\mathbb{N}}$ such that

(i) $t \subseteq t^{\prime} \Rightarrow F(t) \subseteq F\left(t^{\prime}\right)$;

(ii) length $(F(t))=$ length $(t)+1$;

(iii) if $F(\varnothing)=\left(a^{0}, k_{0}\right)$, then $a^{0} \in P$ and for every $c^{0} \in\left[\mathcal{P}_{a^{0}}\right], G\left(\left\langle a^{0}, c^{0}\right\rangle\right)(0)=k_{0}$; if $F\left(m_{0}, \ldots, m_{n}\right)=\left(a^{0}, k_{0}, a^{1}, k_{1}, \ldots, a^{n+1}, k_{n+1}\right)$, then

(a) $\forall i \leq n+1\left(a^{i} \in P\right)$; 
(b) for any $c^{n+1} \in\left[\mathcal{P}_{a^{n+1}}\right]$, if $c^{0}, c^{1}, \ldots, c^{n}$ are the uniquely determined members of $\left[\mathcal{P}_{a^{0}}\right], \ldots,\left[\mathcal{P}_{a^{n}}\right]$ such that

$$
\left(a^{i}\right)^{*}\left(c^{i}\right)=\left\langle\bar{m}_{i}, a^{i+1}, c^{i+1}\right\rangle, \quad i \leq n,
$$

where $\bar{m}_{i}=11 \ldots 1011 \ldots$, with 0 at the $m_{i}$ th place, then $G\left(\left\langle a^{0}, c^{0}\right\rangle\right)(i)=k_{i}$, $\forall i \leq n+1$.

Assuming such $F$ can be found, put $f\left(\left(m_{i}\right)\right)=\left(k_{i}\right)$, so that $f$ is continuous (actually Lipschitz). We claim that

$$
\left(m_{i}\right) \in B \Leftrightarrow f\left(\left(m_{i}\right)\right) \in A,
$$

which completes the proof.

To prove $(*)$, let $\left(a^{0}, a^{1}, \ldots\right)$ be produced by $F$ applied to $\left(m_{0}, m_{1}, \ldots\right)$. For each $n$, define perfect sets $C_{0}^{n}, C_{1}^{n}, \ldots, C_{n}^{n} \subseteq 2^{\mathbb{N}}$ with $C_{i}^{n} \subseteq \mathcal{P}_{a^{i}}$, for $i \leq n$, as follows:

$C_{n}^{n}=\left\{c^{n} \in\left[\mathcal{P}_{a^{n}}\right]:\left(a^{n}\right)^{*}\left(c^{n}\right)=\left\langle\bar{m}_{n}, a^{n+1}, x\right\rangle\right.$, for some $\left.x\right\}$,

$C_{n-1}^{n}=\left\{c^{n-1} \in\left[\mathcal{P}_{a^{n-1}}\right]:\left(a^{n-1}\right)^{*}\left(c^{n-1}\right)=\left\langle\bar{m}_{n-1}, a^{n}, x\right\rangle\right.$, for some $\left.x \in C_{n}^{n}\right\}$,

$C_{0}^{n}=\left\{c^{0} \in\left[\mathcal{P}_{a^{0}}\right]:\left(a^{0}\right)^{*}\left(c^{0}\right)=\left\langle\bar{m}_{0}, a^{1}, x\right\rangle\right.$ for some $\left.x \in C_{1}^{n}\right\}$.

Then we have:

(iv) $c^{0} \in C_{0}^{n} \Rightarrow\left\langle a^{i}, c^{i}\right\rangle$ is good for $i \leq n$, where $c^{1}, \ldots, c^{n}$ are computed according to the formula in (iii)(b).

(v) $n^{\prime} \geq n \Rightarrow C_{i}^{n^{\prime}} \subseteq C_{i}^{n}$ for $i \leq n$.

So $\left[\mathcal{P}_{a^{0}}\right] \supseteq C_{0}^{0} \supseteq C_{0}^{1} \supseteq C_{0}^{2} \subseteq \ldots$ Thus $\bigcap_{n} C_{0}^{n} \neq \varnothing$. Pick $c^{0} \in C_{0}^{n}$, for all $n$. Then all $\left\langle a^{i}, c^{i}\right\rangle$ are good, and $\left(a^{i}\right)^{*}\left(c^{i}\right)=\left\langle\bar{m}_{i}, a^{i+1}, c^{i+1}\right\rangle$ for all $i$, so by (iii)(b) above $G\left(\left\langle a^{0}, c^{0}\right\rangle\right)(i)=k_{i}$ for all $i$. Since

$$
\left\langle a^{0}, c^{0}\right\rangle \in B^{*} \Leftrightarrow G\left(\left\langle a^{0}, c^{0}\right\rangle\right) \in A,
$$

this means that

$$
\forall i\left(\left\langle a^{i}, c^{i}\right\rangle \text { is good }\right) \&\left(m_{i}\right) \in B \Leftrightarrow\left(k_{i}\right) \in A,
$$

or, as $\forall i\left(\left\langle a^{i}, c^{i}\right\rangle\right.$ is good $)$,

$$
\left(m_{i}\right) \in B \Leftrightarrow f\left(\left(m_{i}\right)\right)=\left(k_{i}\right) \in A .
$$

It remains to construct $F\left(m_{0}, \ldots, m_{n}\right)$, and this is done by induction on $n$. To define $F(\varnothing)=\left(a^{0}, k_{0}\right)$, let $H_{0}(a, c)=G(\langle a, c\rangle)(0)$. $H_{0}$ is Borel, so by the niceness of the coding system, we can find $a^{0} \in P$ and $k_{0} \in \mathbb{N}$ with $G\left(\left\langle a, c^{0}\right\rangle\right)(0)=k_{0}$ for all $c^{0} \in\left[\mathcal{P}_{a^{0}}\right]$. Now assume $n>0$ and $F\left(m_{0}, \ldots, m_{n-1}\right)=\left(a^{0}, k_{0}, a^{1}, k_{1}, \ldots, a^{n}, k_{n}\right)$ is given. Define $H_{n+1}: 2^{\mathbb{N}} \times 2^{\mathbb{N}} \rightarrow \mathbb{N}$ as follows: Given $(a, c) \in 2^{\mathbb{N}} \times 2^{\mathbb{N}}$, let $c^{0}, \ldots, c^{n}$ be the unique elements of $\left[\mathcal{P}_{a^{0}}\right], \ldots,\left[\mathcal{P}_{a^{n}}\right]$ resp., such that $\left(a^{i}\right)^{*}\left(c^{i}\right)=\left\langle\bar{m}_{i}, a^{i+1}, c^{i+1}\right\rangle$ for $i<n$ and $\left(a^{n}\right)^{*}\left(c^{n}\right)=\left\langle\bar{m}_{n}, a, c\right\rangle$. Put $H_{n+1}(a, c)=G\left(\left\langle a^{0}, c^{0}\right\rangle\right)(n+1)$. Clearly $H_{n+1}$ is Borel and so, by the niceness of the coding system, we can find $a^{n+1}, k_{n+1}$ such that for all $c^{n+1} \in\left[\mathcal{P}_{a^{n+1}}\right], H_{n+1}\left(a^{n+1}, c^{n+1}\right)=k_{n+1}$. Put

$$
F\left(m_{0}, \ldots, m_{n}\right)=F\left(m_{0}, \ldots, m_{n-1}\right)^{\wedge}\left(a^{n+1}, k_{n+1}\right) .
$$

Remarks. (i) By taking complements, it immediately follows (with the obvious definitions) that a subset of a Polish space is $\boldsymbol{\Sigma}_{1}^{1}$-hard (resp., complete) iff it is Borel $\boldsymbol{\Sigma}_{1}^{1}$-hard (resp., complete).

(ii) Denote the class of $\boldsymbol{\Delta}_{2 n+1}^{1}$ functions by $D_{2 n+1}^{1}$. Then the preceding proof generalizes in a straightforward fashion to show that, assuming $\boldsymbol{\Delta}_{2 n}^{1}$-determinacy, 
a subset of a Polish space is $\Pi_{2 n+1}^{1}$-hard (resp., complete) iff it is $D_{2 n+1}^{1} \Pi_{2 n+1}^{1}$-hard (resp., complete). Similarly for $\Sigma_{2 n+1}^{1}$.

\section{REFERENCES}

1. L. Harrington and A. S. Kechris, On the determinacy of games on ordinals, Ann. Math. Logic 20 (1981), 109-154. MR 83c:03044

2. A. S. Kechris, Classical Descriptive Set Theory, Graduate Texts in Math., vol. 156, SpringerVerlag, 1995.

Department of Mathematics 253-37, California Institute of Technology, Pasadena, CALIFORNia 91125

E-mail address: kechris@math.caltech.edu 